\title{
Quasi-periodic VLF emissions observed during daytime at a low latitude Indian ground station Jammu
}

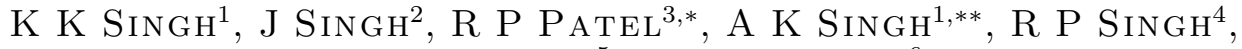 \\ REJESH SINGH ${ }^{5}$ and P A GANAI \\ ${ }^{1}$ Atmospheric Research Laboratory, Department of Physics, Banaras Hindu University, \\ Varanasi 221 005, India. \\ ${ }^{2}$ Department of Physics, G.G.M. Science College, Canal Road, Jammu 180 002, India. \\ ${ }^{3}$ Department of Physics, M.M.H.P.G. College, Ghaziabad, India. \\ ${ }^{4}$ V.K.S. University, Ara 802 301, Bihar, India. \\ ${ }^{5}$ Indian Institute of Geomagnetism, New Panvel, Navi Mumbai 410 218, India. \\ ${ }^{6}$ Department of Physics, National Institute of Technology, Srinagar 190 006, Kashmir, \\ India. \\ *e-mail: rppatel12@yahoo.co.in \\ **e-mail: abhay_s@rediffmail.com
}

This paper reports quasi-periodic pulsing hiss emissions recorded during daytime in the frequency range of $50 \mathrm{~Hz}-15 \mathrm{kHz}$ at low latitude station Jammu (geomag. lat. $=22^{\circ} 26^{\prime} \mathrm{N} ; L=1.17$ ). It is noted that pulsing VLF emissions are a rare phenomena at low latitudes. The various spectrograms of pulsing VLF hiss emissions presented in this paper clearly show band limited spectrums regularly pulsing with almost equal period of the order of few seconds in the frequency range of $\sim 3-8 \mathrm{kHz}$. Generation and propagation mechanism of these emissions are briefly discussed.

\section{Introduction}

This paper is concerned with the most dramatic type of quasi-periodic (QP) emission 'pulsing hiss' in the frequency range $50 \mathrm{~Hz}-15 \mathrm{kHz}$ of magnetospherically generated VLF emissions, as observed at a low latitude ground station Jammu $(L=1.17)$, together with their possible generation mechanisms. The name 'pulsing hiss' seemed more appropriate as the observed sample spectrograms appeared more to resemble band-limited thermal or fluctuation noise, irregularly pulsing with almost equal period (Ward et al 1982). The wave particle interaction occurring in the magnetosphere generates a variety of emissions in the ELF/VLF range. Amplitude-modulated ELF/VLF emissions observed on the ground were classified by Helliwell (1965), who noted various types of periodic emissions, which usually had periods of a few seconds and were often associated with whistler mode waves echoing along geomagnetically field-aligned paths between opposite hemispheres. By contrast, QP emissions consisted of repeated noise bursts of longer (tens of seconds) and more irregular period. QP emissions were further classified into type-I and type-II by Sato et al (1974) on the basis of whether or not they were correlated with geomagnetic pulsations. Hiss-type emissions have been reported which show a degree of periodicity of somewhat shorter periods (from fractions of a second to several seconds), such as 'hisslers' (Ungstrup and Carpenter 1974; Singh et al 2004) and 'pulsing hiss' (Ward et al 1982; Singh et al 2005). These are largely a night phenomenon associated with sub-storm and pulsating aurora respectively (Smith et al 1998). The subject has been reviewed by Sazhin and Hayakawa (1994).

Keywords. Quasi-periodic pulsing hiss; VLF hiss; waveguide mode. 
Although the broadband ELF/VLF hiss emissions are often observed at low latitude ground stations in Japan and India (Hayakawa et al 1975; Khosa et al 1981; Singh et al 1999), there is almost no evidence of the occurrence of pulsing hiss emissions at low latitudes during daytime. An understanding of the generation mechanism of these pulsing VLF hiss emissions observed during daytime at our low latitude ground station Jammu would be most useful for inferring the properties of high energy trapped electrons. With this aim, we have preformed detailed signal analyses. The purpose of this paper is to provide a preliminary description of the most dramatic repeated noise bursts type of pulsed VLF hiss emissions in the sonogram display recorded at a low latitude ground station Jammu. The results reported here are based on the VLF data collected at our ground-based Indian station Jammu during the years from 1996 to 2006.

\section{Experimental results}

The present study is based on the VLF observations made at our newly setup ground station Jammu in India from December 1996 to December 2006. Pulsing ELF/VLF hiss emissions recorded at Jammu are of good quality, and the number of emissions are large enough to be of statistical significance. Broadband VLF signals are received by a T-type antenna, amplifiers and tape recorder having bandwidth of $50 \mathrm{~Hz}-12 \mathrm{kHz}$. T-type antenna is $25 \mathrm{~m}$ in vertical length and $6 \mathrm{~m}$ long horizontally and $3.2 \mathrm{~mm}$ in diameter. Its impedance is about $1 \mathrm{M} \Omega$. The antenna is rendered aperiodic with the help of a suitable R-C network to avoid any possible ringing effect. The antenna is erected at a suitable distance from the main building to reduce the power line hum and any other type of man-made noises. Between the antenna and pre/main amplifiers, an active filtering unit is introduced to reduce the local noise to a minimum in the frequency range $100-500 \mathrm{~Hz}$. The filter is constructed from a suitable $\mathrm{R}-\mathrm{C}$ network along with operational amplifier to be operated in positive feedback mode. The lower cut-off frequency of the filter is about $60 \mathrm{~Hz}$ and voltage gain is $1.2 \mathrm{up}$ to $15 \mathrm{kHz}$. In this recording setup, we have not used anti-aliasing filter. The gain of the pre/main amplifier is varied from 0 to $40 \mathrm{~dB}$ to avoid overloading of the amplifier at the time of great VLF activity. The observations were taken continuously both during day and night-times. The VLF data were stored on the magnetic tapes, which were analyzed using a digital sonograph. Digitization of the analog signal was carried out at $16 \mathrm{kHz}$ sampling frequency. The inbuilt software in the spectrum analysis of the

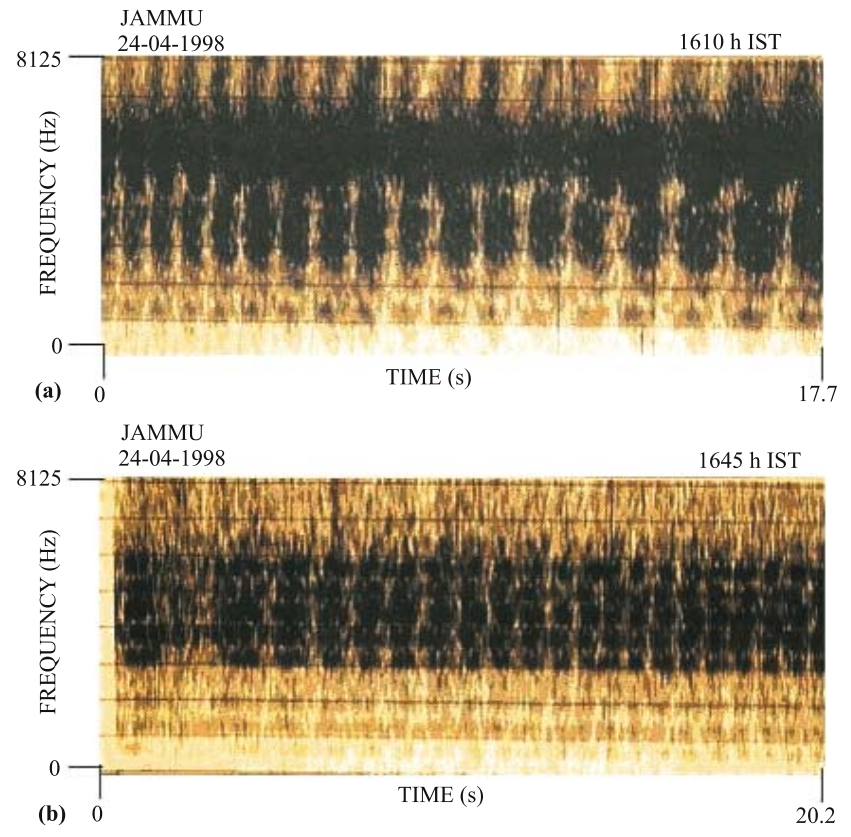

Figure 1. Typical examples of pulsing hiss recorded at Jammu during daytime (a) on 24 April 1998 at 16:10 h IST and (b) on 24 April 1998 at 16:45 h IST.

sonograph machine provides dynamic spectrum, which updates in real time typically covering $8 \mathrm{kHz}$ in frequency and $2.54 \mathrm{~s}$ in time. The frequency range may be varied from $100 \mathrm{~Hz}$ to $40 \mathrm{kHz}$. Among the VLF data acquired during the span of about ten years of recording, we could only get burst type of pulsing ELF/VLF hiss emissions in the years 1998-2003 during day hours. Attempts had been made to record such type of emissions at other Indian stations and Singh et al (2005) have reported observation of pulsing hiss at Varanasi only in night-times. The observation of pulsing hiss emissions at the ground station Jammu during daytime is rare in the sense that most of the reported observations are mainly from $\mathrm{mid} / \mathrm{high}$ latitudes (Ward et al 1982; Smith et al 1998).

Typical examples of pulsing VLF hiss emissions observed during daytime are shown in figures 1-5, which are easily recognizable on frequency time spectrograms. Figure 1 shows typical examples of pulsing VLF hiss emission observed on April 24, 1998 during daytime in the VLF band in the frequency range of about $3-6 \mathrm{kHz}$. The day April 24, 1998 correspond to the highly disturbed day with sum of $K_{P}$ indices as $33\left(\Sigma K_{\mathrm{P}}=33\right)$. The events shown in figure $1(a, b)$ were recorded on April 24, 1998 mainly in the late afternoon between 14:00 IST (Indian Standard Time) local time to 17:00 IST (IST $=+5.5 \mathrm{~h}$ UT). Pulsing hiss emissions shown in figure $1(\mathrm{a}, \mathrm{b})$ contain equispaced pulses of very short duration of about $0.5 \mathrm{~s}$ which are non-dispersive discrete rising tones in the frequency range $\sim 3-8 \mathrm{kHz}$. Figure 2 shows very 

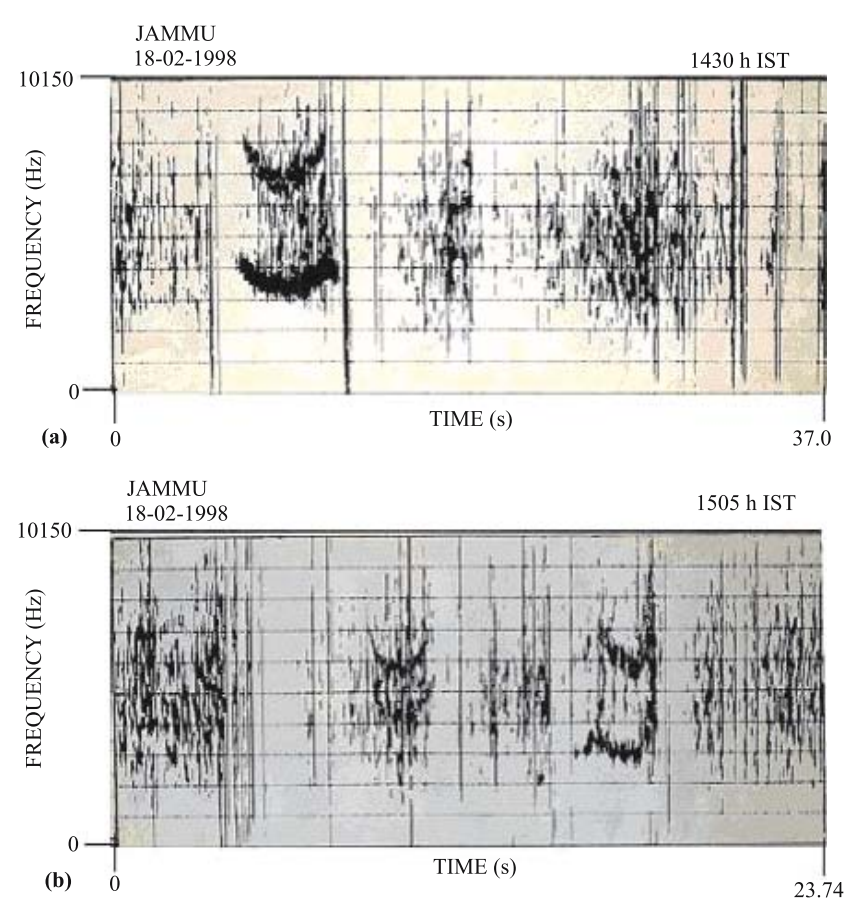

Figure 2. Typical examples of pulsing VLF hiss emission (hyperbolic shape) recorded at Jammu during daytime (a) on 18 February 1998 at 14:30 h IST and (b) on 18 February 1998 at 15:05 h IST.

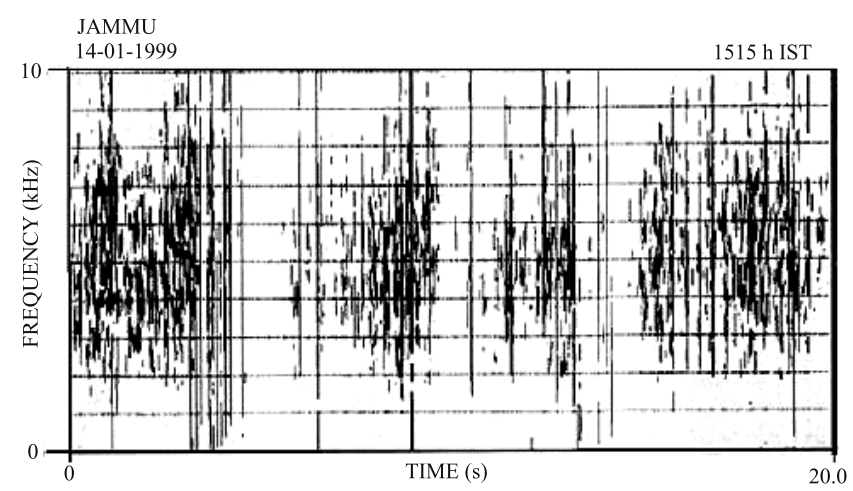

Figure 3. Typical example of pulsing VLF hiss emission of burst type recorded during daytime at Jammu on 14 January 1999 at $13: 15 \mathrm{~h}$ IST.

interesting events of pulsing VLF hiss emission recorded on February 18, 1998 during daytime in which pulses are of well defined spectral shape (hyperbolic shape) and of longer periods. These events were observed on the highly disturbed day February 18, 1998 with sum of $K_{P}$ indices as $33\left(\Sigma \mathrm{K}_{\mathrm{P}}=33_{-}\right)$. Figure $2(\mathrm{a}, \mathrm{b})$ shows two pulses where the hyperbolic arms run out at different angles and different minimum frequencies at the apical points. The frequencies corresponding to the lower arms of hyperbolic pulses in figure 2(a) (14:30 IST) are $4.4-5 \mathrm{kHz}$, with the apical point at a frequency of $3 \mathrm{kHz}$ and occurs for a period of $4.9 \mathrm{~s}$. Whereas the upper hyperbolic pulse corresponds to frequencies $8.1 \mathrm{kHz}$ with the central depression

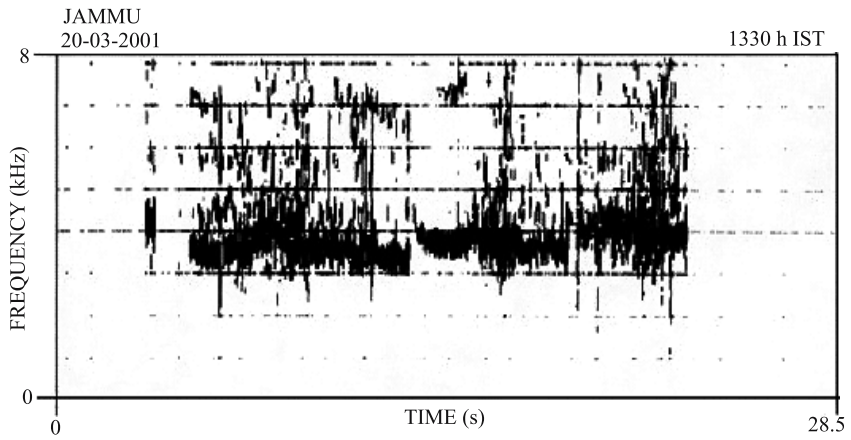

Figure 4. Typical example of pulsing VLF hiss emission of longer period recorded during daytime at Jammu on 20 March 2001 at 13:30 h IST.

at $6.7 \mathrm{kHz}$. The duration of this event is about $4.1 \mathrm{~s}$. Similar but slightly less well-defined hyperbolic pulses are observed in figure 2(b) (15:05 IST) occurring at a frequency stretch of $3.8 \mathrm{kHz}$ (lower band) for duration of $2.12 \mathrm{~s}$ and upper event is observed between frequencies 7.1 and $7.4 \mathrm{kHz}$ for a time interval of $1.8 \mathrm{~s}$.

Figure 3 shows a typical example of pulsing VLF hiss emission of burst type observed on 14 January 1999 during daytime at 15:15 h IST in the frequency range observed on 14 January 1999 during daytime at $15: 15 \mathrm{~h}$ IST local time. The 14 January corresponds to the highly magnetically disturbed day with sum of $\mathrm{K}_{\mathrm{P}}$ indices $31_{+}$ $\left(\Sigma \mathrm{K}_{\mathrm{P}}=31_{+}\right)$. This event was recorded mainly late afternoon between 14:00 h IST and 16:00 h IST. Pulsing hiss emission shown in this figure contains almost equi-spaced burst type of pulses of duration of $\sim 5 \mathrm{~s}$ which are clearly non-dispersion rising tones in the frequency range $\sim 2-8 \mathrm{kHz}$. In figure 4 , we show a very interesting typical example of pulsing VLF hiss emission of diffused type of pulses recorded on 20 March 2001 at 13:30 h IST local time. This event corresponds to highly disturbed day with sum of $\mathrm{K}_{\mathrm{P}}$ indices as $44_{+}\left(\Sigma \mathrm{K}_{\mathrm{P}}=44_{+}\right)$ and was recorded in the early afternoon between 13:00 $\mathrm{h}$ IST and 15:30 $\mathrm{h}$ IST. Pulsing hiss emission shown in this figure contains two diffused traces of duration of about $8 \mathrm{~s}$ which are white noise bands in the frequency $\sim 3-5 \mathrm{kHz}$. Figure 5 illustrates another type of example of pulsing VLF hiss emission recorded on 2 Feburary 2003 at 15:10 h IST local time. This event also corresponds to highly magnetically disturbed day with sum of $K_{P}$ indices $41_{+}\left(\Sigma \mathrm{K}_{\mathrm{P}}=41_{+}\right)$and was recorded in the late afternoon between 14:00 $\mathrm{h}$ IST and 16:00 $\mathrm{h}$ IST. Pulsing hiss emission shown in this figure contains purely equally spaced non-dispersive rising tones observed in the frequency range of $\sim 2-4 \mathrm{kHz}$. The periodicity between each rising tone discrete elements of pulsing hiss emission is $\sim 0.1 \mathrm{~s}$. The pulsing VLF hiss emissions shown in figures 1-5 


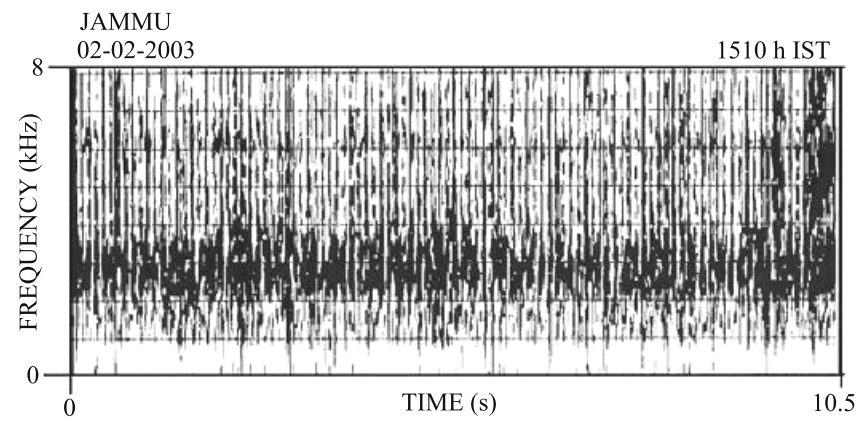

Figure 5. Typical example of pulsing VLF hiss emission recorded at Jammu during daytime on 2 February 2003 at 15:10 h IST.

have been observed during the period of usual normal VLF hiss activity.

From the detailed spectral analysis of the pulsing VLF hiss emissions it is found that the pulsing VLF hiss emissions of repeated noise bursts type are observed in the frequency range of $\sim 3-8 \mathrm{kHz}$. Clearly, the pulses in pulsing VLF hiss emission are mainly undispersed band limited noise consisting of multitude of rising tones of burst type and dispersed signals which on some occasions consist of falling and rising tones of hyperbolic shape. Pulsing VLF hiss emissions reported in this paper both occurred in the late afternoon and peak around 15:00 IST local time during quiet and disturbed days, whereas the ELF pulsing hiss emission observed on GEOS-2 occurred during night-time associated with sub-storms and pulsating aurora (Sazhin and Hayakawa 1994; Smith et al 1998). Pulsing VLF hiss emissions with lower cut-off frequency of $2.5 \mathrm{kHz}$ presented in this paper are observed above the waveguide cut-off frequency. Unfortunately, we do not have the corresponding magnetic pulsation data at our station on those days of observation of pulsing VLF hiss emissions. Sato (1980) has presented several examples of ground-based quasi-periodic hiss emissions with periods in the range $3-10 \mathrm{~s}$ which has good correlation with the corresponding magnetic pulsations. This makes it possible to assume that these magnetic pulsations somehow contribute to the quasi-periodic nature of hiss emissions. This problem was considered in detail by Ward et al (1982) and Ward (1983, 1984), who made a comparative study of pulsing hiss-type events (pulsing hiss) recorded onboard the GEOS-2 satellite in the frequency ranges $0.2-0.6 \mathrm{kHz}$ (filter 1) and $0.6-1.2 \mathrm{kHz}$ (filter 2), pulsing aurora observed with ground-based TV camera and the ground-detected micropulsation. Waves were observed typically at $L=6.5$ in the vicinity of the magnetospheric equatorial plane outside the plasmasphere (Ward 1983). The period of pulsing hiss was usually in the range $2-30 \mathrm{~s}$ with the shorter periods being more common (Ward et al 1982). Ward et al (1982) had attempted to relate different types of pulsing hiss to the corresponding types of pulsations, but they failed to find any convincing correspondence between them, pulsing hiss observations onboard GEOS-2 in the equatorial magnetosphere and simultaneous $2.2 \mathrm{~Hz}$ modulation of auroral electrons observed onboard the rocket were also reported by Lepine et al (1980). Pasmanik et al (2004) have reported the quasi-periodic (QP) ELF/VLF hiss emissions detected onboard the Freja and Magion satellites with an increase in the frequency drift rate during the generation of a single element of QP emission, and an event with alteration of QP elements having different frequency drift rates.

\section{Discussions}

Detailed analysis of the pulsing VLF emissions observed at our low latitude ground station Jammu was made to find out the possibility of their occurrence. The possibility that the occurrence of these pulsing hiss emissions was just a coincidence does not seem to be likely because we have observed many events which occurred one after the other during the same period of normal hiss activity on the same day of observation. We have examined various possibilities for the occurrence of these emissions shown in figures 1-5 which includes (1) that they were caused by the instrument; (2) they were generated due to second order cyclotron resonances and (3) they were caused by HF heating of the lower ionosphere. However, these possibilities are ruled out. We have checked the records of these emissions of those dates mentioned in figures $1-5$ and no evidence of the generation of these emissions due to instrumental effect has obtained because these emissions have been observed during the period of unusual normal VLF hiss activity on those dates. The second order cyclotron resonance requires energetic electrons of $\sim 50 \mathrm{MeV}$ which are not available at $L=1.2$ (Katz 1966), and radiations from HF heating peaks at frequency near $2 \mathrm{kHz}$ which are not observable on the ground if the receiver is away from the source (Singh 1997).

The reception of VLF waves on the Earth's surface clearly shows that the waves may have propagated along the geomagnetic field lines either in ducted mode or in non-ducted pro-longitudinal mode. The source may lie in the equatorial magnetospheric region of low latitudes or in the auroral region. For low latitude source, the path length will be small and hence dispersion of VLF waves would be small. These VLF waves after exiting from the duct propagate through the 
ionosphere along the geomagnetic field line towards the Earth's surface. Due to attenuation of VLF waves the radiated power will be quite small which could not explain the expected maximum wave intensity as experimentally observed at low latitudes (Singh et al 1999). Tsuruda et al (1982) have measured the spatial attenuation of Siple signals and natural chorus emanating from ducts near the Siple conjugate point and showed a relatively rapid decrease of intensity from the foot of the duct $(\sim 7 \mathrm{~dB} / 1000 \mathrm{~km})$ for both Siple signals and chorus. This shows that the attenuation is quite significant for waves propagating through the ionosphere with larger wave normal angles.

It is commonly believed from the study of VLF/ELF emissions observed at low latitudes that they originate in the equatorial magnetosphere of mid/high latitudes and may have propagated along higher $L$-values and after exiting from the duct, they penetrated the ionosphere and are trapped in the Earth-ionosphere waveguide. The wave normal at the entrance into the waveguide is such that they propagated towards the equator and are received at our low latitude ground station Jammu. The upper boundary frequency (UBF) method as developed by Smirnova (1984) has been generally used to find out the location of VLF emissions observed at low latitude ground stations (Singh et al 1996; Babu 1999; Kumar 2000). The upper boundary frequency of the ground observed VLF emissions are determined on the assumption of dipolar geomagnetic field configuration, by the half electron gyrofrequency in the generation region irrespective of the observation station. The $L$-value of the VLF source is then computed with the help of the relation (Smirnova 1984):

$$
L=\left(\frac{440}{f_{U B}}\right)^{1 / 3}
$$

where $f_{U B}$ is upper boundary frequency of the emission in $\mathrm{kHz}$. Such an approach is called UBF method. Making use of equation (1) and the observed parameters, the values of source region of the quasi-periodic pulsing VLF hiss emissions observed at Jammu are found to be around $L=4$.

The origin of pulsing hiss emissions remains unsolved (Sazhin and Hayakawa 1994). The origin of pulsing VLF hiss emissions recorded at low latitude ground station is not clear and hence further experimental studies of these phenomena are needed, before we get a clear phenomenological model, which could be a first step towards a physical model. Therefore, multistation ground measurements, including direction finding at low latitudes would be desirable in order to give a better descriptive model of the pulsing VLF hiss.
The observed features of pulsing VLF hiss emissions recorded during daytime clearly support the view that pulsing VLF hiss events with a lower cutoff $\sim 3 \mathrm{kHz}$ above the waveguide cut-off frequency are generated in the equatorial region of higher $L$-values and reach the lower edge of the ionosphere where they excite the Earth-ionosphere waveguide to propagate towards the equator and are received at low latitude ground station Jammu (Rao et al 1972).

The VLF hiss generated and amplified to observable amplitudes in the equatorial region of higher $L$-values may also propagate in non-ducted mode and reach the equatorial zone of lower $L$-value after magnetospheric reflection. They may also propagate in ducted mode along a lower $L$-value, which can be received at low latitudes (Rycroft 1972; Chang and Inan 1983). The wave normal angle of these waves in the generation region may be quite large. VLF hiss reaching low latitudes by this method will not exhibit a lower frequency cut-off caused by Earth-ionosphere wave guide mode propagation. Thus, in the absence of direction finding measurements, study of dynamic spectra only cannot help us to determine the source of VLF hiss events observed at Jammu.

We now discuss the tentative generation mechanism of quasi-periodic pulsing VLF hiss emissions. The generation mechanism of pulsing VLF hiss observed during daytime at Jammu could be Cerenkov radiation. Recently, Singh et al (1999) have shown that the Cerenkov radiated power at low latitudes is quite small. To explain the observed VLF hiss emissions, the radiated wave should be amplified. They have suggested that the radiated wave may be subsequently amplified by the energetic electron present in the medium through the process of Doppler shifted cyclotron resonance mechanism. Their computations corresponding to low latitudes as well as high latitudes clearly show that Cerenkov radiation mechanism and amplification mechanism through wave-particle interactions are unable to explain the observed amplitudes of VLF hiss. Ward $(1983,1984)$ has studied in detail the morphological characteristics of pulsed ELF hiss observed onboard GEOS-2 satellite. His case studies indicated that pulsed hiss type of ELF emission is a plasma sheet associated phenomenon which depends very critically on the level of the anisotropy in the high energy electrons (Knott and Bahnsen 1981), rather than the increase in intensity of the energetic electrons in the plasma sheet. His case studies further indicated that the Doppler-shifted cyclotron resonance interaction between whistler mode wave and particles available in anisotropic distributions of energetic electrons is involved in the generation of pulsed hiss. Ward et al (1982) first attempted to relate 
different types of pulsing hiss to the corresponding types of pulsations, but they failed to find any convincing correspondence between them. Further, Ward (1983) also paid attention to a certain similarity between chorus spectrograms presented by Tsurutani and Smith (1974) and the corresponding spectrograms of pulsing hiss emissions. But it was not evident to which type, hiss or chorus, the considered phenomenon must be considered.

Pulsing hiss observations onboard GEOS-2 in the equatorial magnetosphere and simultaneous $2.2 \mathrm{~Hz}$ modulation of auroral electrons observed by rocket were also reported by Lepine et al (1980). The problem of origin of pulsing hiss emissions has been studied in detail by Sazhin and Hayakawa (1994) in their review of periodic and quasiperiodic VLF emissions observed at ground-based stations and in the Earth's magnetosphere, where they have pointed out that this problem remains unsolved. A recent paper by Davidson and Chiu (1991) concentrates on the mechanism for auroral pulsations and is only indirectly related to the possible origin of the pulsing hiss. Gorshkov and Gorbunov (1986) reported quasi-periodic emissions at Magadan $(L=2.7)$ whose period decreased from $3.5-4.5$ to $2.5-3.5 \mathrm{~s}$ as the wave frequency increased from 3 to $5 \mathrm{kHz}$. But it is uncertain whether these should be referred to as pulsing hiss (Sazhin and Hayakawa 1994). However, the exact source mechanism remains unknown.

Pulsing hiss observed on the ground may have propagated along a geomagnetic field line either in the ducted or non-ducted mode. The source of energy could be charged particles spiraling along the field lines. Whistler-mode waves propagating along geomagnetic field lines scatter electrons into the loss cone, which may drive highly localized field-aligned currents leading to the generation of Alfvén waves that may set up ULF waves along the field lines. Or these would be generated at higher latitudes, and propagate across L shells. Thus, the equilibrium conditions breakdown when considering such fast variations and the interaction between the waves and the electrons becomes a function of time. However, the condition of resonance interaction remains the same, but the physical parameters involved become a function of time. Singh et al (2005) have reported observation of pulsing hiss in the frequency range $3-12 \mathrm{kHz}$ at low latitude station Varanasi. They proposed that pulsing hiss is generated through Doppler-shifted cyclotron resonance interaction near the geomagnetic equator and propagated to Earth in whistler-mode. Further, ULF waves propagating along the geomagnetic field lines may have modulated the intensity of the emissions resulting into the pulsing hiss. Singh et al (2005) have also computed the growth rate and shown that the growth rate oscillates and the amplitude of the oscillation decreases as $L$-value increases. However, the absolute value of growth rate is larger at larger $L$-values. Davidson and Chiu (1991) have discussed a non-linear mechanism for auroral pulsations, which may provide some more indication on the possible origin of pulsing hiss.

The gyroresonance interactions between whistler mode waves and energetic electrons are an important process in the magnetosphere. Its consequences are the linear wave amplification and generation of new VLF emissions, and also the wave induced pitch angle scattering of magnetospheric particles and the associated particle precipitation into the lower ionosphere (Brice 1964). So the study of pulsing VLF emissions observed at low latitude is one of the best processes to investigate the waveparticle interaction process in the magnetosphere. Further, high energy trapped electrons prove invaluable in assisting the evaluation of several current magnetosphere models, in particular the subject of reconnection of interplanetary geomagnetic filed lines. In this Earth's magnetosphere, whistler made VLF hiss play an important role in the dynamics of radiation belts. Interactions between energetic electron and whistler made waves, in particular, had been suggested as being a primary indicator in determining the morphology of radiation belts (Sonwalkar and Inan 1988). Thus a detailed understanding of the generation, propagation, and maintenance of pulsing VLF hiss emissions observed at low latitudes is an important problem of magnetospheric physics. However, the source of particles that produce quasi-periodic pulsing VLF hiss emissions at low latitudes is not exactly known. It may be solar streams of high energy trapped electrons that are captured by the Earth's field during periods of magnetic disturbances. On the other hand, there is nothing in this new interpretation requiring solar streams. Trapped particles from any source within the Earth's field would do just as well. The detailed discussions on the usefulness of pulsing VLF emissions observed during daytime at Jammu for inferring the properties of high energy trapped electrons are, however, beyond the scope this brief communication.

Thus, from our detailed discussions it is found that the quasi-periodic pulsing VLF hiss emissions observed at Jammu during daytime may be generated in the equatorial magnetosphere of higher $L$-values through a process of Doppler shifted cyclotron mechanism.

\section{Conclusions}

This paper presents daytime observation of pulsing VLF hiss emissions at a low latitude ground station 
showing that pulsing hiss emissions are not limited to mid and high latitudes. Pulsing VLF hiss emissions occurred in the late afternoon and peak around 15:00 h IST local time during disturbed days only. The detailed discussions and the characteristics of pulsing hiss emissions observed during daytime at Jammu show a strong possibility that these are generated in the equatorial region of higher $L$-values through a process of Doppler shifted cyclotron resonance mechanism. But the origin of pulsing VLF hiss observed at low latitude ground station Jammu is far less clear and further experimental studies of these phenomena are needed before we get a clear phenomenological model, which could be the first step towards a physical model. It seems that model suggested by Sazhin (1987) can be used as a starting point for constructing future self-consistent physical models of most types of quasi-periodic emissions. Therefore, further experimental studies are needed in order that we can choose the best approximations for the mathematical model of quasi-periodic pulsing VLF hiss emissions observed at low latitudes.

\section{Acknowledgements}

K K Singh is thankful to CSIR, New Delhi for providing financial assistance in the form of RA. R P Patel is thankful to DST, New Delhi for awarding Fast Track Scheme (SR/FTP/PS-12/2006). The work is partly supported by DST, New Delhi under SERC project and partly by ISRO, Bangalore.

\section{References}

Babu M K 1999 Ionospheric studies by whistlers and VLF emissions at low latitudes; Ph.D. Thesis, B.U. Bhopal.

Brice N M 1964 Discrete VLF emissions from the upper atmosphere, Tech. Rep. No. Sel-64-088, Radio Science Lab., Standford Univ., USA.

Chang H C and Inan U S 1983 A theoretical model study of observed correlations between whistler mode waves and energetic electron precipitation events in the magnetosphere; Geophys. Res. 88 10,053-10,058.

Davidson G T and Chiu Y T 1991 An unusual nonlinear system in the magnetosphere: A possible driver for auroral pulsations; J. Geophys. Res. 96 19,353-19,362.

Gorshkov Yu N and Gorbunov V I 1986 Dispersion characteristics of attenuating quasiperiodic VLF emissions; Geomag. Aeron. 26 881-883.

Hayakawa M, Tanaka Y and Ohtsu J 1975 The morphologies of low latitude and auroral VLF hiss; J. Atmos. Terr. Phys. 37 517-529.

Helliwell R A 1965 Whistler and related ionospheric phenomena (Stanford, California, USA: Stanford University Press).

Katz L 1966 Electron and proton observations; In: Radiation trapped in Earth's magnetic field (ed.) McCormac B M, (Holland: D. Reidel) pp. 129-154.
Khosa P N, Lalmani, Rausaria R R and Ahmed M M 1981 Whistlers and VLF hiss recorded at Srinagar; Ind. J. Radio Space Phys. 10 209-210.

Knott K and Bahnsen A 1981 Rept. on 5th workshop IMS. Obs. in N. Europe.

Kumar R 2000 Probing of inner magnetosphere by low latitude VLF waves, Ph.D. Thesis, B.U. Bhopal.

Lepine D R, Bryant D A and Hall D S 1980 A 2.2 Hz modulation of auroral electrons imposed at the geomagnetic equator; Nature 286 469-471.

Pasmanik D L, Titova E E, Demekhov A G, Trakhtengerts V Y, Santolik O, Jiricek F, Kudela K and Parrot M 2004 Quasi-periodic ELF/VLF wave emissions in the Earth's magnetosphere: Comparison of satellite observations and modeling; Ann. Geophys. 22 4351-4361.

Rao M, Lalmani, Somayajulu V V and Tantry B A P 1972 Dependence of whistler activity on geomagnetic latitude; Ind. J. Radio Space Phys. 1 192-194.

Rycroft M J 1972 VLF emissions in the magnetosphere; Radio Science 7 811-830.

Sato N 1980 Quasi-periodic (QP) ELF-VLF emissions observed in high latitudes; Mem. Natl. Inst. Polar Res. Ser. A., No. 17, Tokyo, Japan.

Sato N, Hayashi K, Kokubun S, Oguti T and Fukunishi H 1974 Relationships between quasi-periodic VLF emission and geomagnetic pulsations; J. Atmos. Terr. Phys. 36 1515-1526.

Sazhin S S 1987 An analytical model of quasi-periodic ELF/VLF emissions; Planet. Space Sci. 35 1267-1274.

Sazhin S S and Hayakawa M 1994 Periodic and quasiperiodic VLF emissions; J. Atmos. Terr. Phys. 56 $735-753$.

Singh B 1997 Middle and high latitude whistlers observed simultaneously in a low latitude station at Agra; J. Geomag. Geoelect. 49 995-1000.

Singh U P, Narayan D, Singh R P and Singh R N 1996 VLF emissions and determination of magnetospheric parameters; Adv. Space Res. 17 (10)105-(10)109.

Singh D K, Singh Ashok K, Patel R P, Singh R P and Singh A K 1999 Two types of ELF hiss observed at Varanasi, India; Ann. Geophys. 17 1260-1267.

Singh, Krishna Kumar, Singh, Rajesh, Singh R P and Shyampati 2004 Hisslers: Quasiperiodic VLF noise forms observed at low latitude ground station Jammu ( $L=1.17)$; Geophys. Res. Lett. 31 L19802-02.

Singh R P, Patel R P, Singh K and Singh A K 2005 Observation of pulsing hiss at low latitudes; J. Atmos. Solar Terr. Phys. 67 1497-1503.

Smirnova N A 1984 Fine structure of the ground observed VLF chorus as an indicator of the wave dust particle interaction process in the magnetosphere; Planet. Space Sci. 32 425-438.

Smith A J, Engebretson M J, Klatt E M, Inan U S, Arnoldy R L and Fukunishi H 1998 Periodic and quasiperiodic ELF/VLF emissions observed by an array of Antarctic stations; J. Geophys. Res. 103 23,611-23,622.

Sonwalkar B S and Inan U S 1988 Wave normal direction and spectral properties of whistler mode hiss observed on the DE-I satellite; J. Geophys. Res. 93 $7493-7514$.

Tsuruda K, Machida S, Terasawa T, Nishida A and Maezaawa K 1982 High spatial attenuation of the Siple transmitter signal and natural VLF chorus observed at ground based chain stations near Roberval, Quebec; J. Geophys. Res. 87742.

Tsurutani B T and Smith E J 1974 Postmidnight chorus: A substorm phenomena; J. Geophys. Res. 79 $118-127$ 
Ungstrup I M and Carpenter D L 1974 Hisslers: Quasiperiodic (T approx. $2 \mathrm{~s}$ ) VLF noise forms at auroral latitudes; J. Geophys. Res. 795196.

Ward I A, Lester M and Thomas R W 1982 Pulsing hiss, pulsating aurora and micropulsations; J. Atmos. Terr. Phys. 44931.
Ward I A 1983 Pulsing hiss and associated phenomena: A morphological study; J. Atmos. Terr. Phys. 45 289-301.

Ward I A 1984 ELF intensity levels at geostationary orbit and pulsating aurora; J. Geophys. Res. $\mathbf{5 5}$ 85-91.

MS received 19 March 2008; revised 25 January 2009; accepted 18 February 2009 